\title{
Cardiovascular primary prevention - directed approach for individual features
}

\author{
Tânia Branco Mano, Sílvia Aguiar Rosa, Pedro Rio, Ana Teresa Timóteo, Rui Cruz Ferreira \\ Department of Cardiology, Hospital de Santa Marta, Centro Hospitalar Universitário Lisboa Central
}

\section{ABSTRACT}

Cardiovascular diseases (CVD) remain the leading cause of morbimortality globally. Despite substantial improvement in outcomes, alarming increases in obesity, diabetes mellitus and other risk factors have been noted in recent years. Despite the majority of CVD being preventable and primary prevention being cost-effective, preventive approaches are poorly implemented in the population at large as well as in individual patients. The pillar of prevention is lifestyle changes (diet, weight, smoking and exercise) followed by, when appropriate, targeting of the main cardiovascular risk factors with pharmacotherapy: lipid lowering therapy, blood-pressure treatment and blood glucose control. Low-dose aspirin as "one-dose-fits-all strategy" remains controversial as primary prevention due to the increased bleeding risk. New scoring systems or further application of imaging techniques (for example, coronary calcium score) are necessary for better risk stratification.

Key words: cardiovascular primary prevention, cardiovascular risk factors, cardiovascular disease, lifestyle changes

\section{INTRODUCTION}

Cardiovascular diseases (CVD) remain the leading cause of disability and mortality globally, although there have been substantial improvements in outcomes in recent decades. ${ }^{1,2}$

Furthermore, the rate of CVD worldwide is predicted to increase as the prevalence of risk factors for CVD rises in previously low-risk countries. $^{3}$

In Portugal, CVD, particularly coronary heart disease (CHD) and stroke, represented $29.7 \%$ of all deaths in 2015 . This was the lowest value achieved in last years due mainly to preventive strategies and improved diagnosis. ${ }^{4}$

However, more recent data shows that the large mortality decline in CVD seen in recent years appears to be dissipating in the United States, which can be extrapolated to other developed countries, and can also reflect an alarming rise in obesity, diabetes mellitus and other risk factors. ${ }^{5}$

The World Health Organisation (WHO) estimates that $80 \%$ of premature CVD is preventable and risk factor amelioration can help reducing the CVD burden. ${ }^{1,6}$ Prevention of CVD, either by employment of lifestyle changes or use of medication, is cost effective, including population-based approaches and actions directed to individuals at risk, but it is still poorly implemented. ${ }^{1}$
In this review the authors aim to discuss current evidence in primary prevention strategies in CVD.

\section{METHODS}

Literature search was performed using the search terms "primary prevention in Cardiovascular Disease", and with a combination of "diet", "exercise", "hypertension", "lipids", "smoking", "aspirin", "statin", "diabetes mellitus" with the term "cardiovascular prevention".

\section{DISCUSSION}

Primary prevention strategies can be achieved by targeting modifiable cardiovascular risk factors in individuals without previous CV event.

Several studies, such as the Framingham and the INTERHEART (Effect of Potentially Modifiable Risk Factors Associated with Myocardial Infarction in 52 Countries) studies investigated risk factors associated with CVD disease and helped to establish the viability of uniform approaches to CVD primary prevention worldwide. ${ }^{7,8}$

These modifiable targets include behavioural (poor diet, smoking, and physical inactivity), environmental and social (air pollution, 


\section{Table 1}

Primary prevention strategies and demonstrated benefits

\begin{tabular}{|c|c|}
\hline Intervention & Approach \\
\hline Diet & $\begin{array}{l}\text { - Low in saturated fatty acids, high in fiber, fruit, vegetables and fish } \\
\text { intake } \\
\text { - Mediterranean-style diet, supplemented with extra-virgin olive oil } \\
\text { or nuts } \\
\text { - "Provegetarian" } \\
\text { - Salt intake }<5 \mathrm{~g} / \text { day }\end{array}$ \\
\hline Exercise & $\begin{array}{l}\text { - At least } 150 \text { minutes a week of moderate aerobic physical activity } \\
\text { or } 75 \text { minutes a week of vigorous aerobic physical activity }\end{array}$ \\
\hline Weight & $\begin{array}{l}\text { - BMI 20-25 achieved with diet, exercise, behaviour modifications, } \\
\text { medications and/or bariatric surgery }\end{array}$ \\
\hline Smoking & $\begin{array}{l}\text { - No expose to tobacco (smoking or second-hand smoke), including } \\
\text { e-cigarettes }\end{array}$ \\
\hline Antiplatelet therapy & - Low-dose aspirin $(75-100 \mathrm{mg})$ is not recommend in primary prevention \\
\hline Lipids & $\begin{array}{l}\text { - LDL-C as primary target, according to CV risk: } \\
<70 \mathrm{mg} / \mathrm{dL} \text { very high-risk patients } \\
<100 \mathrm{mg} / \mathrm{dl} \text { high risk patients } \\
<115 \mathrm{mg} / \mathrm{dl} \text { low-to-moderate risk patients } \\
\text { Achieved with diet and statins and/or ezetimibe and/or PSCK9 } \\
\text { inhibitors } \\
\text { - If Triglycerides }>150 \mathrm{mg} / \mathrm{dl} \text {, besides statin therapy, consider } \\
\text { icosapent ethyl } 4 \mathrm{~g} / \text { day }\end{array}$ \\
\hline Blood glucose & $\begin{array}{l}\text { - HbA1c }<7 \% \text {; }<6.5 \% \text { in selected patients } \\
\text { - Metformin as first-line therapy, followed by SGLT2 inhibitors } \\
\text { (preferable if at risk or with heart failure) or GLP1-Ra if CV risk } \\
\text { factors and/or chronic renal disease present }\end{array}$ \\
\hline Blood pressure (BP) & $\begin{array}{l}-<140 / 90 \mathrm{mmHg} \text { in all patients and, if well tolerated, to }< \\
130 / 80 \mathrm{mmHg}\end{array}$ \\
\hline
\end{tabular}

Benefits
- Reduction of myocardial infarction, stroke, or death from
cardiovascular (CV) causes
- Reversion of coronary artery disease
- Linear relationship between salt reduction, CV events and all-cause
mortality
- Reduction of other CV risk factors (BP, BG)
- Antiatherogenic effects, improve autonomic balance,
cardioprotection against ischemia-reperfusion injury, healthy anti-
inflammatory milieu and ameliorate age-related loss of muscle
mass and strength
- Improvement in BP, low-density lipoprotein cholesterol, triglyceride
and glucose levels
- Reduction in development of atherosclerosis and superimposed
thrombotic phenomena
- Bleeding risk outweigh the reduction of CV events
- Atherosclerotic plaque stabilization, reduction of major
cardiovascular events and pleiotropic effects
- SLGT2i: reduction of heart failure hospitalizations; no reduction in
- - redujor cardiovascular outcomes without established CV disease
long-tions in all major CV events, 10-15\% in all-cause mortality,
nonfatroke, 20\% in coronary events and 40\% in heart failure

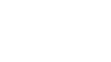

BD - blood pressure; BMI - body mass index; CV - cardiovascular.; DBP - diastolic blood pressure; GLP1-Ra - glucagon-like peptide 1 receptor agonists; PSCK9 - proproteinconvertase subtilisin/kexin type 9; SBP - systolic blood pressure; SGLT2i - selective inhibitors of sodium- glucose cotransporter 2 inhibitors

stress and financial inequalities) and physiological factors such as dyslipidemia, hypertension, and diabetes mellitus.

Here we discuss the main areas targeted for primary prevention of CVD (Table 1.)

\section{Who will benefit from the intervention?}

All current guidelines on CV prevention in clinical practice recommend the assessment of total CVD risk, because atherosclerosis is a product of several risk factors. ${ }^{1,2}$ Estimating the individual 10 -year absolute risk allows matching the intensity of preventive interventions to the patient's absolute risk, to maximize benefit and minimize potential harm from overtreatment. ${ }^{2}$

Since 2003, the European Guidelines have recommended the use of SCORE (Systemic Coronary Risk Estimation) system because it is based on large, representative European cohort datasets. The total $\mathrm{CV}$ risk estimation is recommended for adults $>40$ years of age, unless they are automatically categorised as being at high-risk or very highrisk based on documented CVD, diabetes mellitus ( $>40$ years of age), kidney disease or a highly elevated single risk factor. ${ }^{1}$

However, all risk estimation tools have inherent limitations. In the era of precision medicine, primary prevention strategies are increasingly focusing on refining interventions to match individual patient risk.

Some studies have found underestimation of risk among individuals with chronic inflammatory conditions such as autoimmune diseases $^{9}$ and HIV infection ${ }^{10}$, and those with socioeconomic disadvantage ${ }^{11}$.

On the other hand, people at extremes of ages are not represent in risk scores. Specifically, the elderly population is at greater risk than younger individuals, not only for CVD events but also for adverse outcomes and adverse effects of medications. However, the potential benefits of preventive therapies could be also greater. So, a multidisciplinary evaluation is essential, including factors such as life 
expectancy, time to benefit, physical and cognitive function, comorbidity, medication burden, and frailty. ${ }^{12}$

Therefore, among adults at borderline and intermediate risk, one may consider additional individual risk-enhancing clinical factors to reclassify the risk estimate upward or downward, for example: family history of $C V D^{13}$, chronic inflammatory disease ${ }^{9}$, preeclamp$\mathrm{sia}^{14}$, early menopause ${ }^{15}$, erectile dysfunction ${ }^{16}$, chronic kidney disease, persistently elevated inflammatory markers or elevated lipid biomarkers. If uncertainty remains, US guidelines readdressed this subject in the last recommendations and defended that it is reasonable to measure a coronary artery calcium score to guide clinician-patient risk discussion (class IIa). 2,17

\section{Lifestyle modifications}

\section{Diet}

According to the 2016 Global Burden of Diseases study, the inadequate diet habits of the Portuguese population, namely high salt ingestion, was the main risk factor for loss of disability-adjusted life years. ${ }^{18}$ In Portugal, salt intake has been lowered by setting a saltreduction target for bread, but it remains double the WHO recommendations. ${ }^{19}$

Salt reduction causes a dose-dependent decrease in blood pressure (BP): within the range of 3-12 g daily, the lower the salt intake, the lower the BP. Prospective cohort studies with salt intake measured by multiple 24-h urine collections demonstrate a linear relationship between cardiovascular events and all-cause mortality, down to a daily salt intake of $3 \mathrm{~g}$. It has been demonstrated that, from a public health perspective, salt reduction is one of the most cost- effective, feasible, and affordable measures to prevent CVD. ${ }^{20,21}$

Other recommendations with evidence in reduction CVD risk, although the proportions are not consensual among different guidelines, are diets high in fibre, fruit and vegetable and fish intake, and switching from saturated to polyunsaturated fatty acids. ${ }^{3}$

The Mediterranean-style diet, supplemented with extra-virgin olive oil or nuts, has been consistently shown to reduce major cardiovascular events and is recommend by European guidelines. ${ }^{22} \mathrm{~A}$ "provegetarian" (more vegetables consumption versus animal protein) or vegetarian food pattern (replacing animal protein with plant protein) seems also to reduce mortality rate in small prospective trials, and it has been suggested that it can reverse CHD. ${ }^{23,24,25}$

\section{Exercise and weight}

Exercise is universally recognised as having a positive impact on the majority of health outcomes. Any increase in physical activity reduces the risk of CVD. ${ }^{1,3}$

In addition to merely changing well-known risk factors for systemic CVD, regular exercise can also improve cardiovascular health through non- traditional mechanisms: antiatherogenic effects in the vasculature, improving autonomic balance (thereby reducing the risk of malignant arrhythmias), cardioprotection against ischemia-reperfusion injury and stimulation of myocardial regeneration, promotion of a healthy anti-inflammatory milieu (released by muscle-derived myokines) and ameliorate age-related loss of muscle mass and strength. ${ }^{26}$

Body mass index (BMI) and visceral/central adiposity measured by waist circumference are significant drivers of risk at population level and should be targeted. Maintenance of a healthy weight for reduction of CVD risk by diet, exercise, behaviour modifications, medications and/or bariatric surgery is recommended. Weight loss is associated with moderate improvement in blood pressure (BP), low-density lipoprotein cholesterol, triglyceride and glucose levels. Although the "obesity paradox" is found in established CHD and heart failure, this cannot be misinterpreted in primary prevention because of the influence of physical inactivity. ${ }^{1,2,27}$

\section{Smoking}

Tobacco use is the leading preventable cause of disease, disability and death in developed countries. Almost one-third of CHD deaths are attributable to smoking and second-hand smoke exposure, with smoking cessation the most cost-effective strategy for CVD prevention. $1,2,18,28$. The cardiovascular benefits of quitting are immediate and even among older adults it is beneficial in reducing the excess risk. ${ }^{29}$ Smoking and smoke exposure enhance the development of both atherosclerosis and superimposed thrombotic phenomena, affecting endothelial function, oxidative processes, platelet function, fibrinolysis, inflammation, lipid oxidation and vasomotor function. ${ }^{1}$

Although Electronic Nicotine Delivery Systems ("e-cigarettes") could help in smoking cessation, they are still controversial due to the lack of long-term data. ${ }^{30}$ Caution should be present in their recommendation, since they may increase the risk of cardiovascular and pulmonary diseases. $^{31}$

\section{Medical therapy}

\section{Anti-platelet therapy}

The role of aspirin (acetylsalicylic acid) in inhibiting platelet aggregation has been well established in the secondary prevention of coronary and cerebrovascular diseases. However, its role in primary prevention is still controversial, with conflicting recommendations offered by guidelines. While US guidelines recommend low-dose aspirin (75$100 \mathrm{mg}$ ) among adults between 40 to 70 years who are at higher CVD risk but not at increased bleeding risk, European guidelines do not recommend aspirin for primary prevention. ${ }^{1,2}$

Contrasting with the first randomised studies which support the use of low-dose aspirin to prevent myocardial infarction and stroke, recent evidence suggests less overall benefit of this strategy, with absolute benefits counterbalanced by bleeding risk. ${ }^{32,33,34}$

The ARRIVE (use of Aspirin to Reduce Risk of Initial Vascular Events in patients at moderate risk of cardiovascular disease) trial was a randomised, double-blind, placebo-controlled, multicenter study with 12,546 patients with moderate cardiovascular risk with no diabetes or high risk of bleeding. In this trial, after an average follow-up of 5 years, there were no significant differences between the groups in the primary endpoint (cardiovascular death, myocardial infarction, 
unstable angina, stroke or ischemic attack): $4.3 \%$ vs $4.5 \%$. However the event rate was lower than expected, probably due to contemporary risk strategies management with antihypertensive and lipid-lowering drugs. In line with other trials, there was a nonsignificant $15 \%$ risk reduction in myocardial infarction (MI) in the aspirin group. On the other hand, an increased gastrointestinal bleeding risk was noted in the treatment group (HR 2.11, $p=0.007$ ), without significant difference in all-cause mortality. ${ }^{35}$

Since diabetic patients have higher risk of vascular events, the ASCEND trial (Effects of Aspirin for Primary Prevention in Persons with Diabetes Mellitus) randomized more than 15,000 patients with diabetes and no CVD to aspirin or placebo, for a mean follow-up of 7.4 years. Although aspirin reduced significantly serious vascular events by $12 \%$, the risk of major bleeding was increased by $29 \% .^{36}$ In concordance with this finding, a recent meta-analysis of a total of 13 trials showed significant reduction in cardiovascular outcome $(0.38 \%$ absolute risk reduction, number needed to treat of 265) with an increased risk of major bleeding events $(0.47 \%$ absolute risk increase, number need to harm of 210) with aspirin use. ${ }^{37}$

On the other hand, retrospective meta-analyses of randomized trials of low-dose aspirin have suggested that aspirin may result in a lower risk of cancer, mainly gastrointestinal tract cancer (30 to 40\% risk reduction of colorectal cancer). ${ }^{38,39,40}$ These benefits were not demonstrated in the seven years of follow-up of the ASCEND trial, probably because these effects seem to increase with more prolonged exposure. ${ }^{36}$

One of the reasons suggested for results disparity in aspirin trials is the "one-dose-fits-all strategy", with possible underdosing in patients of large body size and excess dosing in patients of small body size. Obesity and increased BMI are associated with reduced inhibition of cyclo-oxygenase- 1 by low doses of aspirin, probably due to increased platelet activation or turnover and reduced systemic bioavailability. Rothwell and colleagues investigated the effects of the interaction between bodyweight and aspirin dose by combining individual patient data from randomised controlled trials (including nine trials in primary prevention with more than 100 thousand patients). The investigators concluded that the optimal dose of aspirin to prevent cardiovascular events depends on bodyweight: low-dose (75-100 mg) aspirin once a day were ineffective in people weighing $70 \mathrm{~kg}$ or more (particularly in those who smoked or were treated with enteric-coated formulations), whereas higher doses became more effective with increasing weight. These results were also translated in the bleeding, suddendeath and cancer risk, and highlight the potential to improve effectiveness and argue for a more tailored dosing strategy. ${ }^{41}$

The results mentioned above, for the time being, favoured the more conservative management recommend by European Cardiology Society (ESC). Bodyweight should be considered in ongoing and future randomised clinical trials that address aspirin dosing in cardiovascular prevention to clarify how weight-adjusted dosing will affect both benefit and risk.

\section{Lipid and triglyceride lowering therapy}

Hypercholesterolemia is a well-established risk factor for both fatal and non-fatal CV events in individuals with and without previous CVD.
Interventions to ameliorate lipid profile have long been used in primary prevention.

The low-density lipoprotein cholesterol (LDL-C) is the best understood atherogenic subfraction, and an important target for pharmacotherapy. Statins have become the first-choice agents for its reduction. $1,2,3,42$

The earlier statin trials were reported in the 1990s and, since then, several trials have been published highlighting the consistency of treatment benefits across a wide range of patient subgroups. In primary prevention, the large Cholesterol Treatment Trialists (CTT) showed that, per $1.0 \mathrm{mmol} / \mathrm{L}(40 \mathrm{mg} / \mathrm{dL}$ ) LDL-C reduction there is a $10 \%$ proportional reduction in all-cause mortality, $20 \%$ proportional reduction in coronary heart disease, cardiovascular death, $23 \%$ reduction of major coronary events and $17 \%$ reduction of stroke. Other systematic reviews confirmed the dose-dependent reduction in CVD with LDL-C lowering: the greater the $L D L-C$ reduction, the greater the CV risk reduction. Evidence available to date also showed that primary prevention with statins is likely to be cost-effective and may improve patient quality of life. ${ }^{43,44,45,46,47}$

The benefits from statins can be extrapolated even in lower-risk $(<5 \%)$ and older patients ( $>75$ years), often forgotten by clinicians and recommendations. ${ }^{46,48}$

Although the LDL-C reduction is the major effect of statins, in vitro and experimental systems postulate that pleiotropic effect (antiinflammatory and anti-oxidant) can play a significant role in reduction of CV mortality. Nevertheless, this remains controversial and difficult to prove in clinical practice. ${ }^{49,50}$

Since the degree of LDL-C reduction is dose dependent and varies between the different statins and interindividuals, the choice and intensity of statin should be tailored to the patient CV risk and comorbidities (for example, diabetes mellitus). ${ }^{42}$

Equally, the potential side effects of statins were, since their implementation, a matter of debate and non-adherence. In the last Cochrane Review of statins in primary prevention, there was no evidence of any serious harm caused by statin prescription, namely cancer risk. Weak evidence was found for an increased risk of liver enzyme elevations (RR 1.16 - 95\% Cl 0.87 to 1.54), renal dysfunction (RR $1.11,95 \% \mathrm{Cl}$ 0.99 to 1.26 ) and arthritis (RR $1.20-95 \% \mathrm{Cl} 0.82$ to 1.75 ). The main side effect reported was the increased risk of type 2 diabetes in those treated with statins RR 1.18 ( $95 \% \mathrm{Cl} 1.01$ to 1.39$)$. Overall, the absolute reduction in the risk of CVD outweighs the possible adverse effect of a small increase in diabetes incidence, mainly in patients with high and intermediate $\mathrm{CV}$ risk. ${ }^{51}$

Furthermore, the LDL-C reduction benefit is not specific for statin therapy. ${ }^{52}$

In patients intolerant to statins or unable to achieve treatment goals with the maximal recommended dose, ezetimibe, a cholesterol absorption inhibitor, should be used. In a Cochrane review, ezetimibe showed modest beneficial effect on CV endpoints, primarily driven by a reduction in non-fatal myocardial infarction and non-fatal stroke, 
but with limited evidence regarding its role in primary prevention. ${ }^{53}$ The EWTOPIA 75 trial showed recently for the first time that, in elderly Japanese patients with $L D L \geq 140 \mathrm{mg} / \mathrm{dl}$ and $\geq 1$ high-risk feature, the use of ezetimibe in monotherapy for primary prevention reduced CV events (primarily cardiac events), compared with dietary counselling alone. ${ }^{54}$ Even though there is a need for more robust evidence with ezetimibe in primary prevention, its beneficial effect is also supported by genetic studies of Niemann-Pick C1-like protein 1 (the target of ezetimibe): mutations that inactivate the protein were found to be associated with lower plasma LDL-C and lower risk for CHD. ${ }^{55}$

Recently a new class of drugs, proprotein-convertase subtilisin/ kexin type 9 (PCSK9) inhibitors, has become available. This therapeutic strategy uses monoclonal antibodies to inhibit PCSK9, involved in LDL receptors control. PCSK9 inhibitors reduce LDL-C levels by $60 \%$, independent of a background lipid-lowering therapy. Despite the significant reduction in CV events, a decrease in the overall mortality was not achieved, maybe due to the limited follow up of two years. Nevertheless, large trials with longer follow-up are needed to evaluate PCSK9 inhibitors versus active treatments as well as placebo. Owing to the predominant inclusion of very or high-risk patients in these studies (and mostly in secondary prevention), applicability of results to primary prevention is limited. ${ }^{56}$

Based on PCSK9 inhibitors data, it can be postulated that there is no level of LDL-C below which benefit ceases or harm occurs.

On the other hand, some epidemiologic and randomized studies have shown that triglyceride can be an independent risk factor. However, medications that reduce triglyceride levels such as niacin, fibrates, cholesteryl ester transfer protein inhibitors and, until 2018, n-3 fatty acid products, have not been shown to reduce the rates of cardiovascular events when administered in addition to statins. ${ }^{57}$ Only in the last year, the REDUCE-IT trial, a multicenter, randomized, double-blind, placebo-controlled trial with 8179 patients enrolled (30\% of them in primary prevention) showed that a $4 \mathrm{~g} /$ daily dose of icosapent ethyl (a highly purified and stable eicosapentaenoic acid ethyl ester) reduced the primary end point of cardiovascular death, nonfatal myocardial infarction, nonfatal stroke, coronary revascularization and unstable angina by $25 \%$ in patients with hypertriglyceridemia. ${ }^{58}$

\section{Blood glucose control}

Diabetes mellitus is a high prevalent disease and a major cardiovascular risk factor: increasing excess risk two-fold, independently from other conventional risk factors. ${ }^{59}$ In Portugal, the estimate prevalence of diabetes in adults between 20 and 79 years was $13.3 \%$ in 2015 ( 1 in 10 adults) and it is increasing worldwide. ${ }^{60}$ Likewise, CVD remains the leading cause of death and hospitalization in diabetic patients. The high risk for adverse outcomes in this population derives not only from atherosclerotic cardiovascular disease, but also from an increased risk of heart failure independent of coronary disease and renal disease.

Metformin remains the first-line therapy for type 2 diabetes mellitus (T2DM) because of its acceptable safety profile, low cost and efficacy in reducing glycated hemoglobin ( $\mathrm{HbA1c}$ ) and weight. It was in the last decade that the UKPDStudy (United Kingdom Prospective Diabetes Study) showed that intensive therapy with metformin improved outcomes compared to conventional therapy (lifestyle modifications alone): risk reductions of $32 \%$ of diabetes-related endpoint (mainly microvascular and neuropathic complications), 42\% for diabetes-related death, $36 \%$ for all-cause mortality but a nonsignificant trend toward reduced myocardial infarction after 10 years. ${ }^{61}$ More recent trials and meta-analysis concluded that it remains uncertain if metformin significantly reduces risk of CVD, mainly due to absence of evidence, but it is unlikely that a definitive placebo-controlled cardiovascular endpoint trial will be forthcoming. ${ }^{62}$

The intensity of glycemic control (targeting $\mathrm{HbA} 1 \mathrm{c}<6.5 \%$ ) is also a matter of debate. Data suggest that the potential risks of intensive glycemic control may outweigh its benefits (modest reduction in major CVD outcomes) in certain individuals, such as ones with long duration diabetes mellitus, a known history of severe hypoglycemia, advanced atherosclerosis, and a limited life span because of advanced age, frailty, or comorbid conditions. ${ }^{63}$

However, a new paradigm in T2DM glucose treatment has emerged in recent years: choosing the best drug according to the patient's characteristics, namely cardiovascular and/or renal disease. ${ }^{64}$

Since 2008, after the finding that some glucose-lowering drugs, such as rosiglitazone, may be associated with adverse cardiovascular outcomes, the new antihyperglycemic agents must prove cardiovascular safety in randomized trials for their approval by regulatory authorities. These compulsory trials have contributed to a better understanding of the disease over the last decade and, subsequently, two classes of drugs have been shown to reduce CVD in adults with T2DM: selective inhibitors of sodium-glucose cotransporter 2 (SGLT2i), that blocks glucose resorption in the proximal tubule of the kidney and promotes glucosuria, and glucagon-like peptide 1 receptor agonists (GLP-1Ra), that increase insulin and glucagon production in liver, increase glucose uptake in muscle and adipose tissue and decrease hepatic glucose production.

However, there are several considerations that must be taken into account when analysing these studies' data.

First of all, primary prevention patients represent only a relatively small proportion of the study population. The exception was the DECLARETIMI 58 trial with dapaglifozin that evaluated 17,.160 patients, $59.4 \%$ of which had multiple risk factors but without overt CVD. However, the reduction in composite endpoint (myocardial infarction, stroke, and cardiovascular death) did not achieved statistical significance in the dapaglifozin group comparing with placebo in the overall population, or in both subgroups (primary and secondary prevention). ${ }^{65}$

Recent meta-analysis equally concludes that SGLT2i and GLP-1Ra reduce major adverse cardiovascular events (MACE) in a similar magnitude, but restricted to those patients with established CVD in shortterm follow-up ranging from 2 to 4 years. More specifically, GLP1R agonists can reduce the risk of MACE by $12 \%$ (HR $0.88,95 \%-\mathrm{Cl} 0.84$ to $0.94 ; p<0.001$ ) and SGLT2i by $11 \%$ (HR $0.89,95 \%-\mathrm{Cl} 0.83$ to 0.96 ; $\mathrm{p}=0.001)$. Regarding the individual components of MACE, both classes reduced the risk of $\mathrm{MI}(9 \%$ and $11 \%$ ) and CV death ( $12 \%$ and $16 \%)$, but only GLP1-RA reduced the risk of stroke by $14 \% .{ }^{66,67}$ 
Nevertheless, preliminary results from the REWIND trial (Researching CV Events with a Weekly Incretin in Diabetes) with dulaglutide, a GLP-1Ra, seem to show for the first time, in a predominant primary prevention population (69\%), a reduction of major adverse cardiovascular events, during a follow-up period of more than five years. ${ }^{68}$ The final results of this trial and further research comparing these drugs are warranted to explore these findings.

Furthermore, SGLT2i (mostly) and GLP-1Ra have a more robust and consistent effect on the prevention of heart failure and renal outcomes than on atherosclerotic cardiovascular events. In the meta-analysis cited above, GLP1-Ra did not reduce significantly the relative risk of hospitalization for heart failure (HHF) (HR 0.93, p=0.20) whereas SGLT2 reduced the relative risk for HHF by $31 \%(p<0.001)$ and the combined cardiovascular death or HFF by $23 \%(p<0.001)$, regardless of previous atherosclerotic cardiovascular disease or history of heart failure. Likewise, both GLP1-Ra (HR 0.82, p<0.001) and SGLT2i (HR 0.62, p<0.001) reduced the risk of kidney disease progression including macroalbuminuria, but only SGLT2i robustly reduced the risk of worsening Glomerular Filtration Rate (GFR), end-stage kidney disease, or renal death (HR 0.55, p<0.001). The magnitude of benefit of SGLT2i varied with baseline renal function, with greater reduction in hospitalisations for heart failure and lesser reduction in progression of renal disease in patients with more severe kidney disease at baseline. ${ }^{66,67}$

Both drug classes have modest and relatively similar reduction in $\mathrm{HbA} 1 \mathrm{C}$ and therefore appear to exert their beneficial cardiovascular effects independent of glucose control. Besides lowering of blood pressure, both decrease intraglomerular pressure, reduce albuminuria and ameliorate volume overload (for example increasing natriuresis by glycosuria in SGLT2i), so individual pleiotropic properties have been suggested, with possible changes in arterial stiffness, cardiac function and cardiac oxygen demand. ${ }^{69}$

Ongoing trials will assess the effects of SGLT2i in dedicated populations of patients with heart failure with and without diabetes and in diabetic patients with clinical nephropathy.

Keeping these trials in mind, the American Diabetes Association Standard of Medical Care of Diabetes 2019, recommends starting SGLT2 $i$ in patients with T2DM who have established CVD or chronic kidney disease, or GLP1-Ra if HbA1C is below target with metformin. In patients with CVD at high risk of heart failure or in whom heart failure coexists, SGLT2i are preferred. ${ }^{70}$

Interestingly, the 2010 ACC/AHA Guideline on Primary Prevention of Cardiovascular Disease suggested that is reasonable to initiate SGLT2i or GLP1-Ra in patients with T2DM in primary prevention (class of evidence IIb). ${ }^{2}$

However, as part of targeting glucose/glycated hemoglobin (HbA1c), a multifactorial approach is crucial with lifestyle interventions and strict control of blood pressure, cholesterol and other risk factors. ${ }^{1}$

\section{Anti-hypertensive therapies}

Elevated BP was the leading global contributor to premature death in 2015 , with systolic blood pressure (SBP) $\geq 140 \mathrm{mmHg}$ accounting for the majority of the disability burden and mortality, due to ischemic heart disease (4.9 million), hemorrhagic stroke (2.0million), and ischemic stroke (1.5million). ${ }^{71}$

BP has an independent and continuous relationship with the incidence of cardiovascular events (myocardial infarction, cerebrovascular disease, heart failure, atrial fibrillation and peripheral artery disease) and end-stage renal disease. The relationship between BP and risk of events has been shown at all ages and ethnic groups. However, SBP appears to be a better predictor of events than diastolic BP (DBP) after the age of 50 years, probably because DBP tends to decline from midlife as a consequence of arterial stiffening.

The decision to start BP-lowering treatment depends on the BP level, the CV risk (assessed by SCORE system) and the presence of hypertension-mediated organ damage (HMOD). Even asymptomatic HMOD in heart, brain, retina, kidney, and vasculature are independent determinants of cardiovascular risk and can help to reclassify patients, for example identifying high-risk or very high-risk hypertensive patients who may otherwise be misclassified as having a lower risk by the SCORE system. Also, multiorgan damage carries a greater risk than single HMOD. ${ }^{72,73}$ The HMOD with more prognostic value are left ventricular hypertrophy (LVH), chronic kidney disease (CKD) with albuminuria or arterial stiffening. Non-invasive cardiovascular imaging (for example transthoracic echocardiogram, carotid ultrasound or coronary CT) is being increasingly used and continues to provide new technological opportunities to assess this damage at an earlier stage. ${ }^{74}$ Hypertension treatment can induce regression of some manifestations of asymptomatic $\mathrm{HMOD}$, which is associated with a reduction in $\mathrm{CV}$ risk.

In terms of treatment, as previously mentioned, lifestyle interventions are effective (namely dietary salt reduction) but most patients require drug treatment (and the majority a combination of medications). The benefits of treatment are mainly driven by BP reduction per se and not by drug type.

Robust meta-analyses have shown that a $10 \mathrm{mmHg}$ reduction in SBP or a $5 \mathrm{mmHg}$ reduction in DBP is associated with significant reductions in all major CV events by $20 \%$, all-cause mortality by $10-15 \%$, stroke by $\sim 35 \%$, coronary events by $\sim 20 \%$, and heart failure by $\sim 40 \% .{ }^{75}$

Recent data have confirmed that the benefits of antihypertensive treatment have not been attenuated by the widespread concomitant prescription of lipid-lowering and antiplatelet therapies in contemporary medicine. ${ }^{76}$

The protective effect of BP reduction on kidney function can be less obvious and has been restricted to patients with diabetes or CKD, in whom there is a faster rate of disease progression. ${ }^{77}$

All guidelines agree that patients with grade 2 (SBP 160-179mmHg and/or DBP $100-109 \mathrm{mmHg}$ ) or 3 (SBP $\geq 180 \mathrm{mmHg}$ and/or DSB $\geq 110 \mathrm{mmHg}$ ) hypertension should receive antihypertensive drug treatment alongside lifestyle interventions. Recent ESC hypertension guidelines recommend in grade 1 hypertension (SBP 140-159mmHg and/ or DBP $90-99 \mathrm{mmHg}$ ) to initiate immediately drug treatment in patients with elevated risk or HMOD; in low-moderate risk, because of recent trials that have demonstrated significant reduction in all major CV events by BP-lowering drug treatment, drug therapy after 
a trial of lifestyle intervention is now also recommended. In patients with high normal BP, contrasting with 2019 ACC/AHA Guidelines on CVD Primary Prevention, European guidelines still do not advise initiation of drug therapy due to its lack of proven benefit in primary prevention, ${ }^{78,79}$ except perhaps in patients with very high risk, mainly with coronary artery disease. ${ }^{80}$

Highlighting compelling data of intensive lower therapy, the first target of treatment should be to lower BP to $<140 / 90 \mathrm{mmHg}$ in all patients and, if well tolerated, to $130 / 80 \mathrm{mmHg}$ or lower. In older patients (>65 years), SBP should be between 130 and $140 \mathrm{mmHg}$ and DPB to $<80 \mathrm{mmHg}$. Treated SBP should not be targeted to $<120 \mathrm{mmHg}$, because it is associated with increased risk of treatment discontinuation due to adverse events and limited incremental reduction in CV risk.

Specifically in patients with diabetes and $<65$ years, SBP should be targeted to $130 \mathrm{mmHg}$, and until $120 \mathrm{mmHg}$ if tolerated, because of incremental benefit on CV events (mainly stroke). ${ }^{1,79,81,82}$

\section{CONCLUSION}

CVD is multi-factorial and lifestyle changes are the mainstay of any treatment strategy, with patients often requiring behavioural counselling. Those unable to achieve or maintain adequate risk reduction through lifestyle changes alone or those at high risk may benefit from pharmacotherapy: lipid lowering therapy, blood-pressure treatment and blood glucose control, preferably with SGLT2i or GLP-1Ra agents. Considering recent trials, low-dose aspirin as "one-dose-fits-all strategy" continues to play no role in primary prevention due to increased bleeding risk.

It has been suggested that the prescription of a single pill containing a combination of CV drugs (the polypill) could prevent CVD events and be cost effective, in part due to the improvement in adherence to treatment.

In conclusion, it is important to emphasize that more precise risk stratification can be reached by using scoring systems able to incorporate non-traditional risk factors and imaging techniques such as the coronary calcium score.

Disclosure of potential conflicts of interest: none declared.

\section{References}

1. Piepoli MF, Hoes AW, Agewall S, Albus C, Brotons C, Catapano AL, et al. 2016 European Guidelines on cardiovascular disease prevention in clinical practice. European Heart Journal. 2016; 37(29):2315-2381.

2. Arnett DK, Blumenthal RS, Albert MA, Buroker AB, Goldberger ZD, Hahn EJ, et al. 2019 ACC/AHA Guidelines on the Primary Prevention of Cardiovascular Disease. Circulation. 2019

3. Stewart J, Manmathan G, Wilkinson P. Primary prevention of cardiovascular disease: A review of contemporary guidance and literature. Journal of the Royal Society of Medicine Cardiovascular Disease. 2017; 6:1-9.

4. Programa Nacional para as Doenças Cérebro-Vasculares. Programa Nacional para as Doenças Cérebro-Vasculares 2017,Direção Geral da Saúde, Portugal. Available from: https://www.dgs.pt/ portal-da-estatistica-da-saude/diretorio-de-informacao/diretorio-de-informacao/por-serie892489-pdf.aspx?v=11736b14-73e6-4b34-a8e8-d22502108547.
5. McClellan M, Brown N, Califf R, Warner J. Call to Action: Urgent Challenges in Cardiovascular Disease. Circulation. $2019 \mathrm{Feb} ; 139: \mathrm{e} 44-\mathrm{e} 54$.

6. WHO. The challenge of cardiovascular disease - quick statistics. [Online]. [acessed 2019 Abril 14]. Available from: http://www.euro.who.int/en/health-topics/noncommunicable-diseases/cardiovascular-diseases/data-and-statistics.

7. Kannel WB, McGee D, Gordon T. A general cardiovascular risk profile: The Framingham study. The American Journal of Cardiology. 1979 Jul; 38(1): 46-51.

8. Yusuf S, Hawken S, Ounpuu S, Dans T, Avezum A, Lanas F, et al. Effect of potentially modifiable risk factors associated with myocardial infarction in 52 countries (the INTERHEART study): casecontrol study. The Lancet. 2004 Sep; 364(9438):937-52

9. Crowson CS, Gabriel SE, Semb AG, van Riel PLCM, Karpouzas G, Dessein PH, et al. Rheumatoid arthritis-specific cardiovascular risk scores are not superior to general risk scores: a validation analysis of patients from seven countries. Rheumatology (Oxford). $2017 \mathrm{Jul} ;$ 56(7): 1102-1110.

10. Triant VA, Perez J, Regan S, Massaro JM, Meigs JB, Grinspoon SK, et al. Cardiovascular risk prediction functions underestimate risk in HIV infection. Circulation. 2018 May; 137(21):2203-221

11. Colantonio LD, Richman JS, Carson AP, Lloyd-Jones DM, Howard G, Deng L, et al. Performance of the atherosclerotic cardiovascular disease pooled cohort risk equations by social deprivation status. J Am Heart Assoc. 2017 Mar; 6:e005676.

12. Orkaby A, Rich M. Cardiovascular Screening and Primary Prevention in older adults. Clin Geriatr Med. 2018 Feb. 34(1):81-93

13. Patel J, Al Rifai M, Scheuner MT, Shea S, Blumenthal RS, Nasir K, et al.Basic vs more complex definitions of family history in the prediction of coronary heart disease: the Multi-Ethnic Study of Atherosclerosis. Mayo Clinic Proc. 2018 Set; 93(9):1213-1223.

14. Wu P, Haththotuwa R, Kwok CS, Babu A, Kotronias RA, Rushton C, et al. Preeclampsia and future cardiovascular health: a systematic review and meta-analysis. Circ Cardiovasc Qual Outcomes. 2017 Feb; 10(2). pii: e003497

15. Wellons $M$, Ouyang P, Schreiner PJ, Herrington DM, Vaidya D.Early menopause predicts future coronary heart disease and stroke: the Multi-Ethnic Study of Atherosclerosis. Menopause. 2012 Oct; 19(10):1081-7.

16. Uddin SMI, Mirbolouk M, Dardari Z, Feldman DI, Cainzos-Achirica M, DeFilippis AP, et al. Erectile dysfunction as an independent predictor of future cardiovascular events. Circulation. $2018 \mathrm{Jul}$ 31;138(5):540-542.

17. Mahabadi AA, Möhlenkamp S, Lehmann N, Kälsch H, Dykun I, Pundt N, et al. CAC score improves coronary and CV risk assessment above statin indication by ESC and AHA/ACC primary prevention guidelines. JACC Cardiovasc Imaging. 2017 Feb;10(2):143-153.

18. GBD 2016 Causes of Death Collaborators. Global, regional, and national age-sex specific mortality for 264 causes of death, 1980-2016: a systematic analysis for the Global Burden of Disease Study 2016. Lancet. 2017 Sep 16;390(10100):1151-1210.

19. Polonia J, Martins L, Pinto F, Nazaré J. Prevalence, awareness, treatment and control of hypertension and salt intake in Portugal: changes over a decade. The PHYSA study. Journal of hypertension. 2014 Fev; 32:1211-1221

20. Feng J, MacGregor, Graham A. Role of salt intake in prevention of cardiovascular disease: controversies and challenges. Nature. 2018; 15 (6) 371-377.

21. Cook NR, Cutler JA, Obarzanek E, Buring JE, Rexrode KM, Kumanyika SK, et al. Long term effects of dietary sodium reduction on cardiovascular disease outcomes: observational follow-up of the trials of hypertension prevention (TOHP). BMJ. 2007 Apr 28; 334(7599):885-8

22. Estruch R, Ros E, Salas-Salvadó J, Covas MI, Corella D, Arós F, et al. Primary Prevention of Cardiovascular Disease with a Mediterranean Diet Supplemented with Extra-Virgin Olive Oil or Nuts. The New England Journal of Medicine. 2018, Jun 21;378(25):e34.

23. Martínez-González MA, Sánchez-Tainta A, Corella D, Salas-Salvadó J, Ros E, Arós F, et al.A provegetarian food pattern and reduction in total mortality in the Prevención con Dieta Mediterránea (PREDIMED) study. Am J CLin Nutr. 2014 Jul;100 Suppl 1:320S-8S

24. Tharrey M, Mariotti F, Mashchak A, Barbillon P, Delattre M, Fraser GE. Patterns of plant and animal protein intake are strongly associated with cardiovascular mortality: the Adventist Health Study-2 cohort. Int J Epidemiol. 2018 Oct 1;47(5):1603-1612

25. Esselstyn CB Jr, Gendy G, Doyle J, Golubic M, Roizen MF. A way to reverse CAD? J Fam Pract. 2014 Jul;63(7):356-364b.

26. Fiuza-Luces C, Santos-Lozano A, Joyner M, Carrera-Bastos P, Picazo O, Zugaza JL, et al. Exercise benefits in cardiovascular disease: beyond attenuation of traditional risk factors. Nature Reviews Cardiology. 2018 Dec;15(12):731-743.

27. Ma C, Avenell A, Bolland M, Hudson J, Stewart F, Robertson C, et al. Effects of weight loss interventions for adults who are obese on mortality cardiovascular disease, and cancer: systematic review and meta-analysis. BMJ. 2017 Nov 14;359:j4849.

28. Fischerc F, Kraemer A. Meta-analysis of the association between second-hand smoke exposure and ischaemic heart diseases, COPD and stroke. BMJ Public Health. 2015 Dec 1;15:1202

29. Mons U, Müezzinler A, Gellert C, Schöttker B, Abnet CC, Bobak M, et al. Impact of smoking and smoking cessation on cardiovascular events and mortality among older adults: meta-analysis of individual participant data from prospective cohort studies of the CHANCES consortium. BMJ. 2015 Apr 20;350:h1551

30. Pisinger C, Dossing M. A systematic review of health effects of electronic cigarettes. Prev Med. 2014 Dec;69:248-60.

31. Moheimani RS, Bhetraratana M, Yin F, Peters KM, Gornbein J, Araujo JA, et al. Increased Cardiac Sympathetic Activity and Oxidative Stress in Habitual Electronic Cigarette Users: Implications for Cardiovascular Risk. JAMA Cardiol. 2017 Mar 1;2(3):278-284.

32. Steering Committee of the Physicians' Health Study Research Group. Final report on the aspirin component of the ongoing Physicians' Health Study. NEJM. 1989 Jul 20;321(3):129-35.

33. The Medical Research Council's General Practice Research Framework. Thrombosis prevention trial: randomised trial of low-intensity oral anticoagulation with warfarin and low-dose aspirin in 
the primary prevention of ischaemic heart disease in men at increased risk. Lancet. $1998 \mathrm{Jan}$ 24;351(9098):233-41

34. Hansson L, Zanchetti A, Carruthers SG, Dahlöf B, Elmfeldt D, Julius S, et al. Effects of intensive blood-pressure lowering and low-dose aspirin in patients with hypertension: principal results of the Hypertension Optimal Treatment (HOT) randomised trial. Lancet. 1998 Jun 13;351(9118):175562.

35. Gaziano JM, Brotons C, Coppolecchia R, Cricelli C, Darius H, Gorelick PB, et al. Use of aspirin to reduce risk of initial vascular events in patients at moderate risk of cardiovascular disease (ARRIVE): a randomised, double-blind, placebo-controlled trial. Lancet. 2018 Sep 22;392(10152):1036-1046.

36. ASCEND Study Collaborative Group, Bowman L, Mafham M, Wallendszus K, Stevens W, Buck G, Barton J, et al. Effects of Aspirin for Primary Prevention in Persons with Diabetes Mellitus. NEMJ. 2018 Oct 18;379(16):1529-1539.

37. Zheng S, Roddick A. Association of Aspirin Use for Primary Prevention with Cardiovascular Events and Bleeding Events - A systematic Review and Meta-analysis. JAMA. 2019 Jan 22;321(3):277-287.

38. Rothwell PM, Price JF, Fowkes FG, Zanchetti A, Roncaglioni MC, Tognoni G, et al. Short-term effects of daily aspirin on cancer incidence, mortality, and non-vascular death: analysis of the time course of risks and benefits in 51 randomised controlled trials. Lancet. 2012 Apr 28;379(9826):1602-12.

39. Rothwell PM, Wilson M, Elwin CE, Norrving B, Algra A, Warlow CP, et al. Long-term effect of aspirin on colorectal cancer incidence and mortality: 20-year follow-up of five randomised trials. Lancet. 2010 Nov 20;376(9754):1741-50.

40. Rothwell PM, Wilson M, Price J, Belch J, Meade T, Mehta Z. Effect of daily aspirin on risk of cancer metastasis: a study of incident cancers during randomised controlled trials. Lancet. $2012 \mathrm{Apr}$ 28;379(9826):1591-601.

41. Rothwell PM, Cook NR, Gaziano JM, Price JF, Belch JFF, Roncaglioni MC, et al. Effects of aspirin on risks of vascular events and cancer according to bodyweight and dose: analysis of individual patient data from randomised trials. Lancet. 2018 Aug 4;392(10145):387-399.

42. The Task Force for the Management of Dyslipidaemias of the European Society of Cardiology (ESC) and European Atherosclerosis Society. 2016 ESC/EAS Guidelines for the Management of Dyslipidaemias. European Heart Journal. 2016 Aug; 37(39): 2999-3058

43. Mills E, Rachlis B, Wu P, Devereaux P, Arora P, Perri D. Primary prevention of cardiovascular mortality and events with statin treatments: a network meta-analysis involving more than 65,000 patients. JACC. 2008 Nov 25;52(22):1769-81.

44. Brugts JJ, Yetgin T, Hoeks SE, Gotto AM, Shepherd J, Westendorp RG. The benefits of statins in people without established cardiovascular disease but with cardiovascular risk factors: metaanalysis of randomised controlled trials. BMJ. 2009 Jun 30;338:b2376.

45. Cholesterol Treatment Trialists' (CTT) Collaboration, Baigent C, Blackwell L, Emberson J, Holland LE, Reith C, Bhala N, et al. Efficacy and safety of more intensive lowering of LDL cholesterol: a meta-analysis of data from 170,000 participants in 26 randomised trials. Lancet. 2010 Nov 13;376(9753):1670-81

46. Cholesterol Treatment Trialists' (CTT) Collaborators, Mihaylova B, Emberson J, Blackwell L, Keech A, Simes J, Barnes EH, et al. The effects of lowering LDL cholesterol with statin therapy in people at low risk of vascular disease: meta-analysis of individual data from 27 randomised trials. Lancet. 2012 Aug 11;380(9841):581-90.

47. Cholesterol Treatment Trialists' (CTT) Collaboration, Fulcher J, O'Connell R, Voysey M, Emberson J, Blackwell L, Mihaylova B, et al. Efficacy and safety of LDL-lowering therapy among men and women: meta-analysis of individual data from 174,000 participants in 27 randomised trials. Lancet. 2015 Apr 11;385(9976):1397-405

48. Cholesterol Treatment Trialists' Collaboration. Efficacy and safety of statin therapy in older people: a meta-analysis of individual participant data from 28 randomised controlled trials. Lancet. 2019 Feb 2;393(10170):407-415

49. Davignon J. Beneficial cardiovascular pleiotropic effects of statins. Circulation. 2014 Jun 15;109(23 Suppl 1):III39-43.

50. Zhou Q, Liao J. Pleiotropic effects of statins. Basic research and clinical perspectives. Circulation J. May; $74(5): 818-26$

51. Taylor F, Huffman MD, Macedo AF, Moore TH, Burke M, Davey Smith G, et al. Statins for the primary prevention of cardiovascular disease (Review). Cochrane Database of Systematic Reviews. $2013 \operatorname{Jan} 31 ;(1): C D 004816$.

52. Silverman MG, Ference BA, Im K, Wiviott SD, Giugliano RP, Grundy SM, et al. Association Between Lowering LDL-C and Cardiovascular Risk Reduction Among Different Therapeutic Interventions. JAMA. 2016 Sep 27;316(12):1289-97.

53. Zhan S, Tang M, Liu F, Xia P, Shu M, Wu X. Ezetimibe for the prevention of cardiovascular disease and all cause mortality events (Review). Cochrane Database of Systematic Reviews. 2018 Nov 19;11:CD012502

54. Ouchi Y. Ezetimibe in Prevention of Cerebro- and Cardiovascular Events in Middle- to High-Risk, Elderly (75 Years Old or Over) Patients With Elevated LDL-Cholesterol - EWTOPIA 75. In American Heart Association Annual Scientific Sessions (AHA 2018); 2018; Chicago.

55. Myocardial Infarction Genetics Consortium Investigator, Stitziel NO, Won HH, Morrison AC, Peloso GM, Do R, Lange LA, et al. Inactivating mutations in NPC1L1 and protection from coronary heart disease. NEJM. 2014 Nov 27:371(22):2072-82

56. Schmidt A, Pearce L, Wilkins J, Overington J, Hingorani A, Casas J. PCSK9 monoclonal antibodies for the primary and secondary prevention of cardiovascular disease. Cochrane Database of Systematic Reviews. 2017 Apr 28;4:CD011748.

57. Triglyceride Coronary Disease Genetics Consortium and Emerging Risk Factors Collaboration, Sarwar N, Sandhu MS, Ricketts SL, Butterworth AS, Di Angelantonio E, Boekholdt SM, et al. Triglyceride-mediated pathways and coronary disease: collaborative analysis of 101 studies. Lancet. 2010 May 8;375(9726):1634-9.

58. Bhatt DL, Steg PG, Miller M, Brinton EA, Jacobson TA, Ketchum SB, et al. Cardiovascular Risk Reduction with Icosapent Ethyl for Hypertriglyceridemia. NEJM. 2019 Jan 3;380(1):11-22.
59. Sarwar N, Gao P, Seshasai S, Gobin R, Kaptoge S, Di A, et al. Diabetes mellitus, fasting blood glucose concentration, and risk of vascular disease: a collaborative meta-analysis of 102 prospective studies. Lancet. 2010 Jun 26;375(9733):2215-22.

60. Sociedade Portuguesa de Diabetologia. Diabetes: factos e números - 0 ano de 2015 - Relatório anual do Observatório Nacional. 2016.

61. UK Prospective Diabetes Study Group. Effect of intensive blood-glucose control with metformin on complications in overweight patients with type 2 diabetes (UKPDS 34). UK Prospective Diabetes Study (UKPDS) Group. Lancet. 1998 Sep 12;352(9131):854-65.

62. Griffin S, Leaver J, Irving G. Impact of metformin on cardiovascular disease: a meta-analysis of randomised trials among people with type 2 diabetes. Diabetologia. 2017 Sep;60(9):1620-1629.

63. Fox C, Golden S, Anderson C, Bray G, Burke L, Boer I, et al. Update on Prevention of Cardiovascular Disease in Adults With Type 2 Diabetes Mellitus in Light of Recent Evidence: A Scientific Statement From the American Heart Association and the American Diabetes Association. Diabetes Care. 2015 Sep;38(9):1777-803.

64. Aguiar C, Duarte R, Carvalho D. Nova abordagem para o tratamento da diabetes: da glicemia à doenc, a cardiovascular. Revista Portuguesa de Cardiologia. 2019 Jan; 38(1):53-63.

65. Wiviott S, Raz I, Bonaca M, Mosenzon O, Kato E, Cahn A, et al. Dapagliflozin and Cardiovascular Outcomes in Type 2 Diabetes. NEJM. 2019 Jan 24;380(4):347-357.

66. Zelniker T, Wiviott S, Raz I, Im K, Goodrich E, Furtado R, et al. Comparison of the Effects of Glucagon-Like Peptide Receptor Agonists and Sodium-Glucose Cotransporter 2 Inhibitors for Prevention of Major Adverse Cardiovascular and Renal Outcomes in Type 2 Diabetes Mellitus. Circulation. 2019 Apr 23;139(17):2022-2031.

67. Zelniker T, Wiviott S, Raz I, Im K, Goodrich L, Bonaca M, et al. SGLT2 inhibitors for primary and secondary prevention of cardiovascular and renal outcomes in type 2 diabetes: a systematic review and meta-analysis of cardiovascular outcome trial. Lancet. 2019 Jan 5;393(10166):31-39.

68. Press Release. Trulicity (dulaglutide) demonstrates superiority in reduction of cardiovascular events for broad range of people with type 2 diabetes. https://investor.lilly.com/news-releases/ news-release-details/trulicityr-dulaglutide-demonstrates-superiority-reduction. [Online]. [cited 20190514

69. Verma S, McMurray J. SGLT2 inhibitors and mechanisms of cardiovascular benefit: a state-of-theart review. Diabetologia. 2018 Oct;61(10):2108-2117

70. American Diabetes Association. Standards of Medical Care in Diabetes-2019. Diabetes Care 2019. 2019;: p. 42(Supplement 1): S1-S2.

71. Forouzanfar M, Liu P, Roth G, Ng M, Biryukov S, Marczak L, et al. Global burden of hypertension and systolic blood pressure of at least 110 to $115 \mathrm{~mm} \mathrm{Hg}, 1990-2015$. JAMA. $2017 \mathrm{Jan}$ 10;317(2):165-182

72. The Task Force for the management of arterial hypertension, Williams B, Mancia G, Spiering W, Agabiti Rosei E, Azizi M, Burnier M, et al. 2018 ESC/ESH Guidelines for the management of arterial hypertension. European Heart Journal. 2018 Sep 1;39(33):3021-3104

73. Volpe M, Battistoni A, Tocci G, Rosei E, Catapano E, Coppo R, et al. Cardiovascular risk assessment beyond Systemic Coronary Risk Estimation: a role for organ damage markers. J Hypertens. 2012 Jun;30(6):1056-64.

74. Perrone-Filardi P, Coca A, Galderisi M, Paolillo S, Alpendurada F, de Simone G, et al. Noninvasive cardiovascular imaging for evaluating subclinical target organ damage in hypertensive patients: a consensus article from the European Association of Cardiovascular Imaging, the European Society of Cardiology Council on Hypertension and the Eur. J Hypertens. 2017 Sep;35(9):1727-1741.

75. Ettehad D, Emdin C, Kiran A, Anderson S, Callender T, Emberson J, et al. Blood pressure lowering for prevention of cardiovascular disease and death: a systematic review and meta-analysis. Lancet. 2016 Mar 5;387(10022):957-967.

76. Collins R, MacMahon S. Blood pressure, antihypertensive drug treatment and the risks of stroke and of coronary heart disease. Br Med Bull. 1994 Apr;50(2):272-98

77. Lv J E, Sarnak M, Tighiouart H, Jun M, Ninomiya T, Foote C, et al. Effects of intensive blood pressure lowering on the progression of chronic kidney disease: a systematic review and meta-analysis. CMAJ. 2013 Aug 6;185(11):949-57

78. Thomopoulos C, Parati G, Zanchetti A. Effects in individuals with high-normal and normal blood pressure: overview and meta-analyses of randomized trials. J HYpertens. 2017 Nov;35(11):21502160.

79. Wright JJ, Williamson J, Whelton P, Snyder J, Sink K, Rocco M, et al. A Randomized Trial of Intensive versus Standard Blood-Pressure Control. NEJM. 2015 Nov 26;373(22):2103-16

80. Brunström M, Carlbelg B. Association of Blood Pressure Lowering With Mortality and Cardiovascular Disease Across Blood Pressure Levels: A Systematic Review and Meta-analysis. JAMA Intern Med.. 2018 Jan 1;178(1):28-36.

81. Lonn E, Bosch J, López-Jaramillo P, Zhu J, Liu L, Pais P, et al. Blood-Pressure Lowering in Intermediate-Risk Persons without Cardiovascular Disease. NEJM. 2016 May 26;374(21):2009-20

82. Margolis K, O'Connor P, Morgan T, Buse J, Cohen R, Cushman W, et al. Outcomes of combined cardiovascular risk factor management strategies in type 2 diabetes: the ACCORD randomized trial. Diabetes care. 2014 Jun;37(6):1721-8.

\section{Correspondence to:}

Sílvia Aguiar Rosa, MD

Department of Cardiology, Hospital de Santa Marta, Centro Hospitalar Universitário Lisboa Central.

Rua de Santa Marta, no 50, 1169-024, Lisbon, Portugal.

E-mail: silviaguiarosa@gmail.com 